\title{
ON THE CHEMISTRY OF HYDRIDES OF N ATOMS AND O ${ }^{+}$IONS
}

\author{
Zainab Awad $^{1,2}$, Serena Viti ${ }^{2}$, and David A. Williams ${ }^{2}$ \\ ${ }^{1}$ Astronomy, Space Science, \& Meteorology Department, Faculty of Science, Cairo University, Giza, Egypt; zma@sci.cu.edu.eg \\ ${ }^{2}$ Physics and Astronomy Department, University College London, Gower Street, London WC1E 6BT, UK \\ Received 2015 November 5; revised 2016 January 28; accepted 2016 May 23; published 2016 August 1
}

\begin{abstract}
Previous work by various authors has suggested that the detection by Herschel/HIFI of nitrogen hydrides along the low-density lines of sight toward G10.6-0.4 (W31C) cannot be accounted for by gas-phase chemical models. In this paper we investigate the role of surface reactions on dust grains in diffuse regions, and we find that formation of the hydrides by surface reactions on dust grains with efficiency comparable to that for $\mathrm{H}_{2}$ formation reconciles models with observations of nitrogen hydrides. However, similar surface reactions do not contribute significantly to the hydrides of $\mathrm{O}^{+}$ions detected by Herschel/HIFI that are present along many sight lines in the Galaxy. The $\mathrm{O}^{+}$hydrides can be accounted for by conventional gas-phase chemistry either in diffuse clouds of very low density with normal cosmic-ray fluxes or in somewhat denser diffuse clouds with high cosmic-ray fluxes. Hydride chemistry in dense dark clouds appears to be dominated by gas-phase ion-molecule reactions.
\end{abstract}

Key words: astrochemistry - dust, extinction - ISM: abundances - ISM: clouds - ISM: molecules

\section{INTRODUCTION}

Observations using Herschel/HIFI have been made of rotational lines of hydrides $\mathrm{NH}, \mathrm{NH}_{2}$, and $\mathrm{NH}_{3}$ of nitrogen atoms toward G10.6-0.4 (Persson et al. 2010) and of the hydrides $\mathrm{OH}^{+}, \mathrm{H}_{2} \mathrm{O}^{+}$, and $\mathrm{H}_{3} \mathrm{O}^{+}$of oxygen ions toward the same object (Gerin et al. 2010) and toward Orion KL (Gupta et al. 2010). The nitrogen hydrides have also been observed toward W49 (Persson et al. 2012) and toward the Class 0 protostar IRAS 16293-2422 (Hily-Blant et al. 2010). The hydrides of oxygen ions, $\mathrm{O}^{+}$, have been studied along 20 lines of sight and in many velocity components toward bright submillimeter continuum sources in the Galaxy (Indriolo et al. 2015). Hydrides not directly associated with stellar sources are generally believed to be present in gas of relatively low-density along the line of sight.

These detections have stimulated a re-examination of the chemistry of hydrides in both diffuse (e.g., Hollenbach et al. 2012; Indriolo et al. 2015) and dark (Dislaire et al. 2012; Le Gal et al. 2014a, 2014b) interstellar clouds. Both gas-phase and grain-surface reactions have been invoked in these studies.

In this paper, we focus mainly on diffuse gas because of its relative simplicity. We re-examine the predictions of hydride abundances from diffuse cloud models based on gas-phase chemistries to which surface reactions may also contribute. In particular, we study the sensitivity of the chemistry to variations in the physical parameters that control the chemistry. We shall examine first the chemistry of the nitrogen hydrides, and shall conclude, along with other authors, that gas-phase schemes are incapable of supplying the abundances implied by the observations of nitrogen hydrides in diffuse gas. However, grain-surface reactions with plausible efficiencies are capable of supplying nitrogen hydrides in the observed abundances and with the observed ratios of $\mathrm{NH}_{3} / \mathrm{NH}_{2}$ and $\mathrm{NH}_{3} / \mathrm{NH}$. We shall find that the reverse is true for the hydrides of $\mathrm{O}^{+}$, i.e., that reactions of $\mathrm{O}$ atoms with grain surfaces contributing $\mathrm{H}_{2} \mathrm{O}$ molecules to the gas-phase do not significantly affect the $\mathrm{O}^{+}$ hydrides; they do, however, slightly affect the neutral hydrides $\mathrm{OH}$ and $\mathrm{H}_{2} \mathrm{O}$.

\section{SURFACE CHEMISTRY}

Hydride formation in surface reactions was first discussed over half a century ago (McCrea \& McNally 1960). Pickles \& Williams (1977) explored an extensive nitrogen chemistry in a "gas-phase + grain surface" reaction model. Very detailed studies of gas + grain chemistry in diffuse clouds on sight lines toward $\zeta$ Per and $\zeta$ Oph were made by Wagenblast et al. (1993). In particular, a good match for more than 30 atomic and molecular abundances toward $\zeta$ Per was obtained, including $\mathrm{NH}$, for which the column density had been measured by Meyer \& Roth (1991). The NH column density predicted by Wagenblast et al. (1993) for the $\zeta$ Oph model was later confirmed by observations with ultrahigh resolution (Crawford \& Williams 1997). Gas-phase models fail to supply enough $\mathrm{NH}$ for these lines of sight, so these computations may be regarded as strong support for a contribution to interstellar chemistry from surface reactions in diffuse clouds.

Several experimental studies have been made of sequential hydrogenation of nitrogen atoms in various matrices and ice analogues (Hiraoka et al. 1995; Hidaka et al. 2011; Fedoseev et al. 2015; see also the review by Linnartz et al. 2015). These studies confirm that this hydrogenation is efficient and probably barrier-free even at the low temperatures of interstellar dust grains. A detailed description of the process is not possible because of the very limited data on binding energies, mobilities, and desorption processes of the various species involved. In this work, we adopt the version of surface chemistry of Wagenblast \& Williams (1996), in which a neutral nitrogen or oxygen atom arriving at a grain surface is converted directly to $\mathrm{NH}_{3}$ or $\mathrm{H}_{2} \mathrm{O}$ and ejected promptly into the gas; the desorption is assumed to be driven by energies released in the reaction. The reaction is assumed to occur with the same efficiency as the $\mathrm{H}_{2}$ formation reaction, but the arrival rate of $\mathrm{N}$ or $\mathrm{O}$ atoms at grain surfaces is reduced by the square root of the atomic mass of the atom, and, of course, by the abundance of atoms relative to hydrogen (all values of which are taken from Asplund et al. (2009), who give the fractional abundances of $\mathrm{N}$ and $\mathrm{O}$ relative to hydrogen as $6.761 \times 10^{-5}$ and $4.898 \times 10^{-4}$, respectively). 
The $\mathrm{H}_{2}$ formation rate adopted in our computations is $1.3 \times 10^{-17} T^{1 / 2} n(\mathrm{H}) n_{\mathrm{H}}$ in $\mathrm{cm}^{-3} \mathrm{~s}^{-1}$ (e.g., Jura 1974; Tielens 2005). Similarly, the rate of formation of hydrides of $\mathrm{N}$ and $\mathrm{O}$ can be expressed, in $\mathrm{cm}^{-3} \mathrm{~s}^{-1}$, as $3.5 \times 10^{-18} T^{1 / 2} n$ (N) $n_{\mathrm{H}}$ and $3.3 \times 10^{-18} T^{1 / 2} n(\mathrm{O}) n_{\mathrm{H}}$; where $T$ is the gas kinetic temperature, $n(\mathrm{X})$ is the number density of free $\mathrm{X}$ atoms, and $n_{\mathrm{H}}$ is that of hydrogen in any form.

\section{CLOUD MODEL AND GAS-PHASE CHEMISTRY}

The cloud geometry is a one-dimensional slab of uniform density, illuminated by the interstellar radiation field (RF) from one side. The reference model (hereafter, RM) has $n_{\mathrm{H}}=100 \mathrm{~cm}^{-3}$, is illuminated by the Draine interstellar RF, here denoted by $G_{0}$, and irradiated by cosmic-rays (CR) that cause hydrogen ionization at a rate $\zeta_{\text {ISM }}=1.3 \times 10^{-17} \mathrm{~s}^{-1}$ (Wakelam et al. 2005). The gas to dust ratio is assumed to be uniform and such that one visual magnitude of extinction is equivalent to $1.6 \times 10^{21}$ total $\mathrm{H} \mathrm{cm}^{-2}$ (Draine 2011). The model results described below are normally for a cloud of one visual magnitude.

The gas-phase chemistry that leads to the formation of nitrogen hydrides in dense dark clouds has been reviewed by Le Gal et al. (2014b). In these clouds, nitrogen is mainly in $\mathrm{N}$ atoms and $\mathrm{N}_{2}$ molecules. Exchange reactions of $\mathrm{N}$ and $\mathrm{N}_{2}$ with $\mathrm{H}_{2}$ molecules are strongly suppressed at the low temperatures in diffuse and dark clouds. Hence, these neutral routes with hydrogen molecules do not proceed. The reaction of $\mathrm{N}$ atoms with $\mathrm{H}_{3}^{+}$ions (created by cosmic-ray ionization of $\mathrm{H}_{2}$ ) has a high activation energy; in fact, the reverse reaction $\mathrm{H}_{2}+\mathrm{NH}^{+} \longrightarrow \mathrm{H}_{3}^{+}+\mathrm{N}$ is a fast proton transfer. The product $\mathrm{N}_{2} \mathrm{H}^{+}$formed in the proton transfer reaction $\mathrm{N}_{2}+\mathrm{H}_{3}^{+} \longrightarrow \mathrm{N}_{2} \mathrm{H}^{+}+\mathrm{H}_{2}$ has a minor channel of dissociative recombination leading to $\mathrm{NH}$ that is important in dense dark clouds, but is unimportant in diffuse clouds because $\mathrm{N}_{2}$ is a very minor component in them. For example, Knauth et al. (2004), using far-UV absorption spectroscopy of matter in diffuse clouds toward HD 124314, were able to show that only about $10^{-4}$ of nitrogen was in the form of $\mathrm{N}_{2}$.

In diffuse clouds the main route to form $\mathrm{NH}^{+}$, and thence neutral nitrogen hydrides by subsequent hydrogen abstraction reactions and dissociative recombinations, is $\mathrm{N}^{+}+$ $\mathrm{H}_{2} \longrightarrow \mathrm{NH}^{+}+\mathrm{H}$, where the $\mathrm{N}^{+}$ion is formed by cosmicray ionization of $\mathrm{N}$ atoms. This rate coefficient, including its dependence on the $\mathrm{H}_{2}$ ortho/para ratio, has been the subject of several theoretical and experimental investigations (e.g., Marquette et al. 1988; Le Bourlot 1991; Gerlich 1993; Dislaire et al. 2012; Zymak et al. 2013). The experimental results of Zymak et al. confirm the rate coefficient of Dislaire et al. However, the role of fine-structure excitation in $\mathrm{N}^{+}$ions in the reaction may require further investigation. We adopt the value of rate coefficient for the $\mathrm{N}^{+}+\mathrm{H}_{2}$ reaction given in the $\mathrm{UDfA}^{3}$ database (McElroy et al. 2013). At $100 \mathrm{~K}$, this differs from that of Zymak et al. by a factor of three or less, and from the value in the KIDA database by a factor of less than two. The rate coefficients adopted for the main reactions determining nitrogen hydrides are shown in Table 1.

The gas-phase chemistry that leads to the formation of hydrides of $\mathrm{O}^{+}$ions has been reviewed recently by Hollenbach et al. (2012) and by Indriolo et al. (2015). In their Figure 1, Hollenbach et al. summarize the two main channels to the ions

\footnotetext{
3 Also known as UMIST; http://udfa.ajmarkwick.net/.
}

$\mathrm{OH}^{+}, \mathrm{H}_{2} \mathrm{O}^{+}$, and $\mathrm{H}_{3} \mathrm{O}^{+}$. The first of these is initiated by the cosmic-ray ionization of atomic hydrogen, followed by the accidental resonance charge exchange of the hydrogen ion with an oxygen atom, forming $\mathrm{O}^{+}$, which then successively abstracts $\mathrm{H}$ from $\mathrm{H}_{2}$ to form $\mathrm{OH}^{+}, \mathrm{H}_{2} \mathrm{O}^{+}$, and $\mathrm{H}_{3} \mathrm{O}^{+}$. Dissociative recombination of these ions leads to neutral products $\mathrm{O}, \mathrm{OH}$, and $\mathrm{H}_{2} \mathrm{O}$. All these reactions must compete with photoprocesses and other reactions. This first channel is significant in diffuse clouds, where both atomic and molecular hydrogen are abundant.

The second channel involves the reaction of $\mathrm{O}$ atoms with $\mathrm{H}_{3}^{+}$ions arising from the cosmic-ray ionization of $\mathrm{H}_{2}$. The reaction generates $\mathrm{OH}^{+}$and $\mathrm{H}_{2} \mathrm{O}^{+}$ions, which, as in the first channel, abstract hydrogen from $\mathrm{H}_{2}$ molecules to form the full set of $\mathrm{O}^{+}$hydrides. This channel is likely to be dominant in denser regions of the interstellar medium. A sketch of the main gas-phase entry chemical reactions in our model to form hydrides of $\mathrm{N}, \mathrm{O}$, and $\mathrm{O}^{+}$is illustrated in Figure 1, and the main reactions are listed in Table 1.

Rate constants are calculated using the mathematical expression described in UMIST database releases (e.g., Millar et al. 1997; Le Teuff et al. 2000; Woodall et al. 2007; McElroy et al. 2013) as follows:

$$
k=\left\{\begin{array}{l}
\text { (a) if two-body reaction } \\
\alpha(T / 300 \mathrm{~K})^{\beta} \exp (-\gamma / T) \mathrm{cm}^{3} \mathrm{~s}^{-1} \\
\text { (b) if CR ionization reaction, for } \zeta=\zeta_{\text {ISM }} \\
\alpha \quad \mathrm{s}^{-1}
\end{array}\right.
$$

where $\zeta$ is the $\mathrm{CR}$ ionization rate, and the constants $\alpha, \beta$, and $\gamma$ are listed in Table 1.

The elements included in the chemistry are $\mathrm{H}, \mathrm{He}, \mathrm{O}, \mathrm{C}, \mathrm{N}$, $\mathrm{S}, \mathrm{Mg}, \mathrm{Si}$, and $\mathrm{Cl}$, and the chemical network includes 158 species interacting in 2132 chemical reactions, whose rate coefficients are taken from the UDfA 2012 database (McElroy et al. 2013). The rate equations for all species are integrated and molecular abundances provided as a function of time at a particular visual extinction, $A_{\mathrm{V}}$. A full description of the chemical model, UCL_CHEM, used in the current work is given in Viti \& Williams (1999), with some updates described in Viti et al. (2004) and Roberts et al. (2007).

\section{NITROGEN HYDRIDES}

Figure 2 shows that the fractional abundances at steady-state of the three nitrogen hydrides for the RM are all larger than $10^{-9}$ when grain-surface reactions contribute, and at least an order of magnitude lower when they are excluded. Figure 2 shows that, in the RM, the fractional abundance of $\mathrm{NH}_{3}$ is less than $10^{-13}$ and probably undetectable, when excluding the grain-surface contribution. These steady-state fractional abundances are-as expected-found to be independent of the cosmic-ray ionization rate (for a range up to 50 times the canonical interstellar value) and also independent of the initial $\mathrm{H}_{2}$ fraction.

The fractional abundances depend on the cloud density; see Figure 3(a). The fractional abundances of nitrogen hydrides are also sensitive to the RF; see Figure 3(b). Note that if surface reactions are excluded, then the pure gas-phase chemistry generates fractional abundances of nitrogen hydrides that are approximately proportional to the cosmic-ray ionization rate. 
Table 1

The Main Gas-phase Chemical Reactions in Our Network with Their Rate Constants.

\begin{tabular}{|c|c|c|c|c|c|c|c|c|c|c|c|}
\hline \multicolumn{9}{|c|}{ Chemical Reactions } & \multirow{2}{*}{$\begin{array}{c}\alpha \\
1.20 \mathrm{E}-17\end{array}$} & \multirow{2}{*}{$\frac{\beta}{0.00}$} & \multirow{2}{*}{$\begin{array}{l}\gamma \\
0.0\end{array}$} \\
\hline $\mathrm{H}_{2}$ & + & $\mathrm{CR}$ & $\rightarrow$ & $\mathrm{H}_{2}^{+}$ & + & $\mathrm{e}^{-}$ & & & & & \\
\hline $\mathrm{H}_{2}^{+}$ & + & $\mathrm{H}_{2}$ & $\rightarrow$ & $\mathrm{H}_{3}^{+}$ & + & $\mathrm{H}$ & & & 2.08E-09 & 0.00 & 0.0 \\
\hline $\mathrm{H}$ & + & $\mathrm{CR}$ & $\rightarrow$ & $\mathrm{H}^{+}$ & + & $\mathrm{e}^{-}$ & & & $5.98 \mathrm{E}-18$ & 0.00 & 0.0 \\
\hline $\mathrm{H}^{+}$ & + & $\mathrm{O}$ & $\rightarrow$ & $\mathrm{O}^{+}$ & + & $\mathrm{H}$ & & & $6.86 \mathrm{E}-10$ & 0.26 & 224.3 \\
\hline $\mathrm{H}$ & + & $\mathrm{O}^{+}$ & $\rightarrow$ & $\mathrm{O}$ & + & $\mathrm{H}^{+}$ & & & $5.66 \mathrm{E}-10$ & 0.36 & -8.6 \\
\hline $\mathrm{H}_{2}$ & + & $\mathrm{O}^{+}$ & $\rightarrow$ & $\mathrm{OH}^{+}$ & + & $\mathrm{H}$ & & & $1.70 \mathrm{E}-09$ & 0.00 & 0.0 \\
\hline $\mathrm{H}_{3}^{+}$ & + & $\mathrm{O}$ & $\rightarrow$ & $\mathrm{OH}^{+}$ & + & $\mathrm{H}_{2}$ & & & $7.98 \mathrm{E}-10$ & -0.16 & 1.4 \\
\hline $\mathrm{OH}^{+}$ & + & $\mathrm{e}^{-}$ & $\rightarrow$ & $\mathrm{O}$ & + & $\mathrm{H}$ & & & $3.75 \mathrm{E}-08$ & -0.50 & 0.0 \\
\hline $\mathrm{H}_{2}$ & + & $\mathrm{OH}^{+}$ & $\rightarrow$ & $\mathrm{H}_{2} \mathrm{O}^{+}$ & + & $\mathrm{H}$ & & & $1.01 \mathrm{E}-09$ & 0.00 & 0.0 \\
\hline $\mathrm{H}_{3}^{+}$ & + & $\mathrm{O}$ & $\rightarrow$ & $\mathrm{H}_{2} \mathrm{O}^{+}$ & + & $\mathrm{H}$ & & & $3.42 \mathrm{E}-10$ & -0.16 & 1.4 \\
\hline $\mathrm{H}_{2} \mathrm{O}^{+}$ & + & $\mathrm{e}^{-}$ & $\rightarrow$ & $\mathrm{OH}$ & + & $\mathrm{H}$ & & & $8.60 \mathrm{E}-08$ & -0.50 & 0.0 \\
\hline $\mathrm{H}_{2}$ & + & $\mathrm{H}_{2} \mathrm{O}^{+}$ & $\rightarrow$ & $\mathrm{H}_{3} \mathrm{O}^{+}$ & + & $\mathrm{H}$ & & & $6.40 \mathrm{E}-10$ & 0.00 & 0.0 \\
\hline $\mathrm{H}_{3} \mathrm{O}^{+}$ & + & $\mathrm{e}^{-}$ & $\rightarrow$ & $\mathrm{H}_{2} \mathrm{O}$ & + & $\mathrm{H}$ & & & 7.09E-08 & -0.50 & 0.0 \\
\hline $\mathrm{H}_{3} \mathrm{O}^{+}$ & + & $\mathrm{e}^{-}$ & $\rightarrow$ & $\mathrm{OH}$ & + & $\mathrm{H}_{2}$ & & & 5.37E-08 & -0.50 & 0.0 \\
\hline $\mathrm{H}_{3} \mathrm{O}^{+}$ & + & $\mathrm{e}^{-}$ & $\rightarrow$ & $\mathrm{OH}$ & + & $\mathrm{H}$ & + & $\mathrm{H}$ & $3.05 \mathrm{E}-07$ & -0.50 & 0.0 \\
\hline $\mathrm{N}$ & + & $\mathrm{CR}$ & $\rightarrow$ & $\mathrm{N}^{+}$ & + & $\mathrm{e}^{-}$ & & & $2.70 \mathrm{E}-17$ & 0.00 & 0.0 \\
\hline $\mathrm{N}^{+}$ & + & $\mathrm{H}_{2}$ & $\rightarrow$ & $\mathrm{NH}^{+}$ & + & $\mathrm{H}$ & & & $1.00 \mathrm{E}-09$ & 0.00 & 85.0 \\
\hline $\mathrm{NH}^{+}$ & + & $\mathrm{H}_{2}$ & $\rightarrow$ & $\mathrm{NH}_{2}^{+}$ & + & $\mathrm{H}$ & & & $1.28 \mathrm{E}-09$ & 0.00 & 0.0 \\
\hline $\mathrm{NH}_{2}^{+}$ & + & $\mathrm{e}^{-}$ & $\rightarrow$ & $\mathrm{NH}$ & + & $\mathrm{H}$ & & & $9.21 \mathrm{E}-08$ & -0.79 & 17.1 \\
\hline $\mathrm{NH}_{2}^{+}$ & + & $\mathrm{H}_{2}$ & $\rightarrow$ & $\mathrm{NH}_{3}^{+}$ & + & $\mathrm{H}$ & & & $2.70 \mathrm{E}-10$ & 0.00 & 0.0 \\
\hline $\mathrm{NH}_{3}^{+}$ & + & $\mathrm{e}^{-}$ & $\rightarrow$ & $\mathrm{NH}$ & + & $\mathrm{H}$ & + & $\mathrm{H}$ & $1.55 \mathrm{E}-07$ & -0.50 & 0.0 \\
\hline $\mathrm{NH}_{3}^{+}$ & + & $\mathrm{e}^{-}$ & $\rightarrow$ & $\mathrm{NH}_{2}$ & + & $\mathrm{H}$ & & & $1.55 \mathrm{E}-07$ & -0.50 & 0.0 \\
\hline $\mathrm{NH}_{3}^{+}$ & + & $\mathrm{H}_{2}$ & $\rightarrow$ & $\mathrm{NH}_{4}^{+}$ & + & $\mathrm{H}$ & & & $3.09 \mathrm{E}-13$ & 1.08 & -50.9 \\
\hline $\mathrm{NH}_{4}^{+}$ & + & $\mathrm{e}^{-}$ & $\rightarrow$ & $\mathrm{NH}_{2}$ & + & $\mathrm{H}_{2}$ & & & 4.72E-08 & -0.60 & 0.0 \\
\hline $\mathrm{NH}_{4}^{+}$ & + & $\mathrm{e}^{-}$ & $\rightarrow$ & $\mathrm{NH}_{2}$ & + & $\mathrm{H}$ & + & $\mathrm{H}$ & $3.77 \mathrm{E}-08$ & -0.60 & 0.0 \\
\hline $\mathrm{NH}_{4}^{+}$ & + & $\mathrm{e}^{-}$ & $\rightarrow$ & $\mathrm{NH}_{3}$ & + & $\mathrm{H}$ & & & $8.49 \mathrm{E}-07$ & -0.60 & 0.0 \\
\hline $\mathrm{NH}_{3}$ & + & $\mathrm{h}+$ & $\rightarrow$ & $\mathrm{NH}_{3}^{+}$ & + & $\mathrm{H}$ & & & $3.70 \mathrm{E}-09$ & -0.50 & 0.0 \\
\hline $\mathrm{NH}_{3}$ & + & $\mathrm{H}_{3}^{+}$ & $\rightarrow$ & $\mathrm{NH}_{4}^{+}$ & + & $\mathrm{H}_{2}$ & & & 4.39E-09 & -0.50 & 0.0 \\
\hline $\mathrm{N}_{2}$ & + & $\mathrm{H}_{3}^{+}$ & $\rightarrow$ & $\mathrm{N}_{2} \mathrm{H}^{+}$ & + & $\mathrm{H}_{2}$ & & & $1.80 \mathrm{E}-09$ & 0.00 & 0.0 \\
\hline $\mathrm{N}_{2} \mathrm{H}^{+}$ & + & $\mathrm{e}^{-}$ & $\rightarrow$ & $\mathrm{N}$ & + & $\mathrm{NH}$ & & & 2.09E-08 & -0.74 & 0.0 \\
\hline $\mathrm{N}_{2}$ & + & $\mathrm{He}^{+}$ & $\rightarrow$ & $\mathrm{N}^{+}$ & + & $\mathrm{N}$ & + & $\mathrm{He}$ & $9.60 \mathrm{E}-10$ & 0.00 & 0.0 \\
\hline
\end{tabular}

Note. The reactions shown are the main gas-phase entry chemical reactions in our network to form hydrides of $\mathrm{N}, \mathrm{O}$, and $\mathrm{O}^{+}$with their rate constants taken from the UMIST 2012 ratefile (McElroy et al. 2013). The rate constants $\left(k, \mathrm{in}^{3} \mathrm{~s}^{-1}\right)$ are calculated from $k=\alpha(T / 300 \mathrm{~K})^{\beta} \exp (-\gamma / T)$.

\section{OXYGEN ATOM AND ION HYDRIDES}

Figure 2 shows results in the RM for all hydrides of $\mathrm{O}$ atoms and $\mathrm{O}^{+}$ions, both when surface reactions contribute and when they do not. There is a significant time-dependence in the chemistry. This arises because the chemistry is driven by slow cosmic-ray ionization, and also because the chemistry is affected by the $\mathrm{H} / \mathrm{H}_{2}$ balance, which takes a long time (several $\times 10^{9} \mathrm{yr} / n_{\mathrm{H}} \mathrm{cm}^{-3}$ ) to reach steady-state in diffuse clouds. In fact, the time to attain steady-state may exceed the lifetime of a diffuse cloud (often taken to be a few million years). In that case, the chemistry of $\mathrm{O}^{+}$ions may never reach a steady state.

Figure 2 indicates a very slight enhancement in the RM fractional abundances of $\mathrm{OH}$ and $\mathrm{H}_{2} \mathrm{O}$ when surface reactions are operating. However, surface reactions do not affect the RM fractional abundances of the $\mathrm{O}^{+}$hydrides either at the peak fractional abundances (at $\sim 10^{5} \mathrm{yr}$ ) or at steady-state. The peak fractional abundances of the ions are of the order of $10^{-10}$ in the RM, while their steady-state values are an order of magnitude lower.

The $\mathrm{O}^{+}$hydrides are found to be fairly insensitive to the RF; this is because their chemistry is dominated by fast ionmolecule reactions. However, the fractional abundances of these hydrides are sensitive to both gas density and cosmic-ray ionization rate, see Figures 4(a) and (b).
Figure 4(a) shows that the fractional abundances of $\mathrm{O}^{+}$ hydrides depend approximately inversely on the density. For example, with $n_{\mathrm{H}}=10 \mathrm{~cm}^{-3}$, peak fractional abundances (i.e., all ion abundances measured at the $\mathrm{OH}^{+}$peak) are larger by an order of magnitude (and arise earlier, at an evolutionary time $\sim 10^{5} \mathrm{yr}$ ) than for $n_{\mathrm{H}}=100 \mathrm{~cm}^{-3}$ (where peak fractional abundances arise at $\sim 10^{6} \mathrm{yr}$ ). Similar statements can be made for the steady-state fractional abundances. The effects of high cosmic-ray ionization rates on peak abundances are shown in Figure 4(b). Abundances are roughly proportional to the ionization rate. Such behavior has been described by Hollenbach et al. (2012) and was obtained in earlier work by Bayet et al. (2011).

\section{DISCUSSION AND CONCLUSIONS}

\subsection{Nitrogen Hydrides in Diffuse Clouds}

It is clear from results from the RM (see Figure 2) that gasphase models fail to provide nitrogen hydrides in the required abundances as measured toward G10.6-0.4 by Persson et al. (2010), who came to the same conclusion. The data of Indriolo et al. (2015) show that this gas is mainly atomic and is therefore quite diffuse. In particular, pure gas-phase models such as that of Le Petit et al. (2004) cannot provide enough $\mathrm{NH}_{3}$ in diffuse clouds to match the $\mathrm{NH}_{3} / \mathrm{NH}$ and $\mathrm{NH}_{3} / \mathrm{NH}_{2}$ ratios obtained from observations (see below). In gas-phase models of low- 


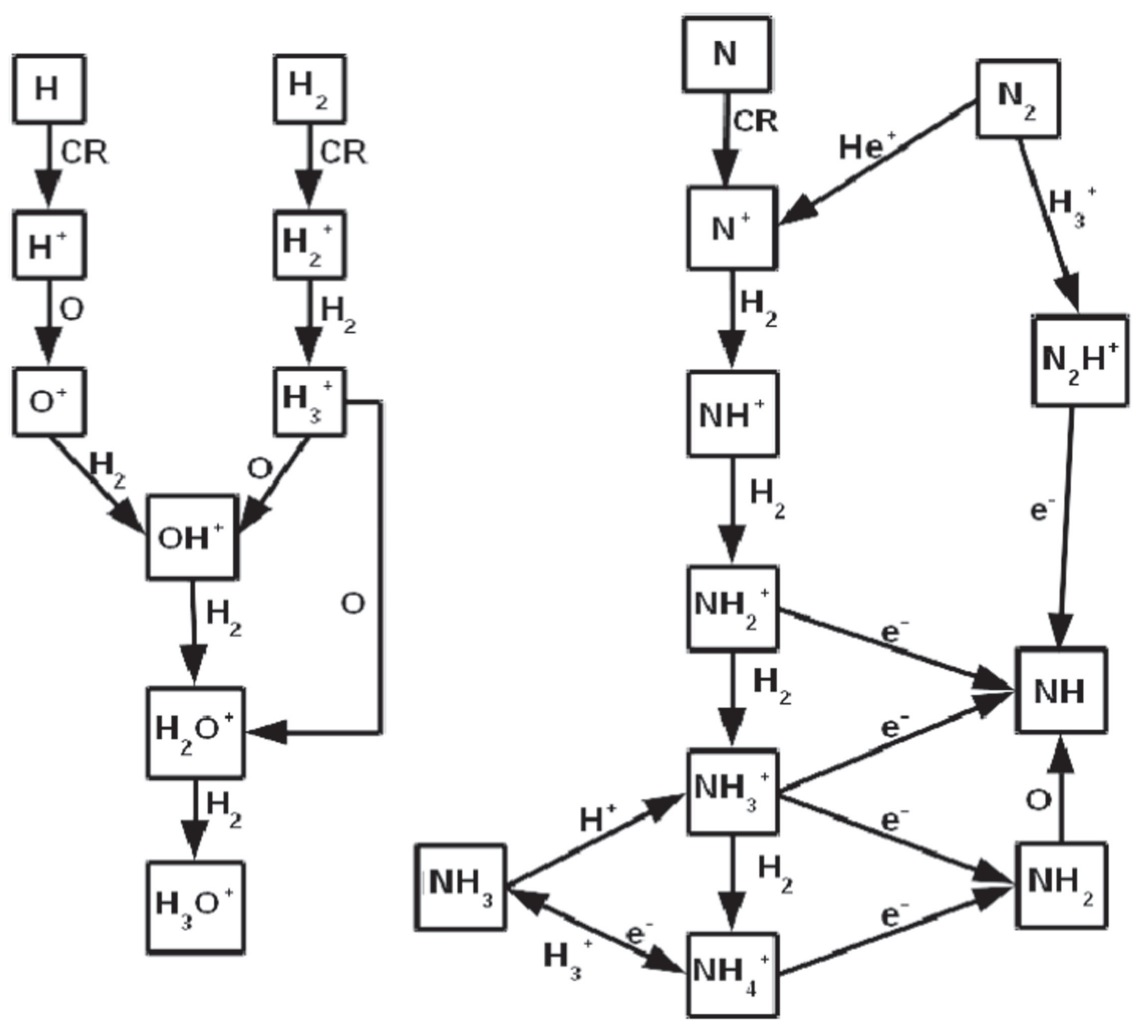

Figure 1. Main routes in the gas-phase chemistry forming hydrides of $\mathrm{N}$ and $\mathrm{O}^{+}$. Diagrams modified from Hollenbach et al. (2012) and Le Gal et al. (2014b).

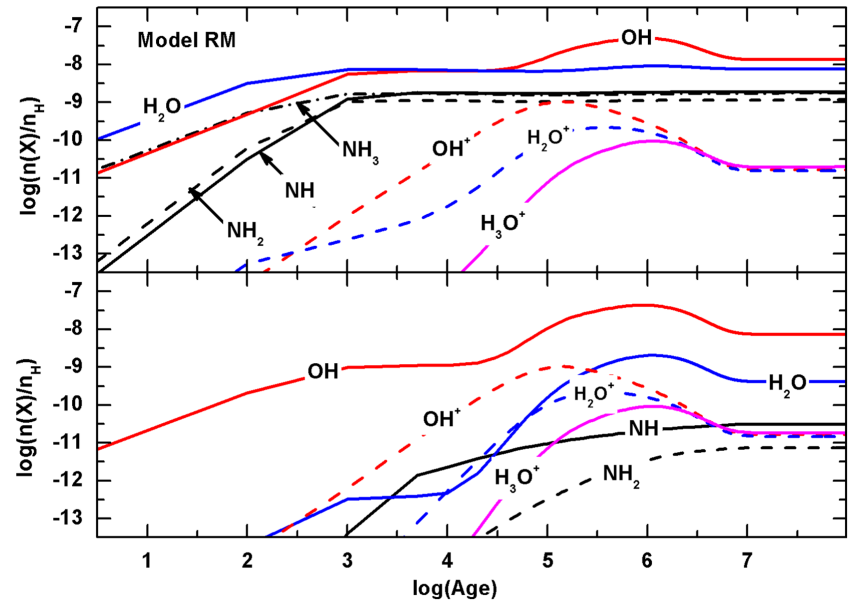

Figure 2. Fractional abundances in the reference model $\left(n_{\mathrm{H}}=100 \mathrm{~cm}^{-3}\right.$, $\zeta=\zeta_{\text {ISM }}, \mathrm{RF}=G_{0}$, but with $\left.\mathrm{H}_{2}(t=0)=0\right)$ showing the time dependence of all the hydrides, with surface reactions included (top) and excluded (bottom). Note that when surface reactions are excluded, the fractional abundance of $\mathrm{NH}_{3}$ is less than $10^{-13}$ (its value at steady-state is $\sim 5 \times 10^{-15}$ ).

density clouds, the predicted ratios fail by several orders of magnitude. However, the contribution from surface reactions can be significant and comparable to the observed fractional abundances. In the (arbitrarily chosen) RM almost the same amount of nitrogen is in the three nitrogen hydrides as is observed to be the case toward G10.6-0.4.

However, the distribution of nitrogen among the three hydrides does not match the observed results toward G10.6-0.4. Persson et al. (2010) found the fractional abundances for $\mathrm{NH}$, $\mathrm{NH}_{2}$, and $\mathrm{NH}_{3}$ to be approximately $(6,3,3) \times 10^{-9}$, respectively. Figures 3(a) and (b) show that the three species respond differently to changes in cloud density and RF. The computed ratios $\mathrm{NH}_{3} / \mathrm{NH}$ and $\mathrm{NH}_{3} / \mathrm{NH}_{2}$ may be compared with the observed values of 0.5 and 1.0 , respectively, see Table 2. Models with low number densities $\left(n_{\mathrm{H}} \sim 30 \mathrm{~cm}^{-3}\right)$ and radiation fields $\left(0.5-1 G_{0}\right)$ can match the observed ratios, but the total amount of nitrogen in all nitrogen hydrides is less than a fifth of that observed. Evidently, we need somewhat higher number densities (say, $n_{\mathrm{H}} \sim 100 \mathrm{~cm}^{-3}$ ) with somewhat reduced $\mathrm{RF}\left(\sim 0.25 G_{0}\right)$ to have the observed total fractional abundance of nitrogen hydrides $\left(\sim 1 \times 10^{-8}\right)$. For the RM, the ratios do not match those observed. Figures 3(a) and (b) show that it is not possible simultaneously to match both high fractional abundances and appropriate ratios of the nitrogen hydrides detected along the line of sight toward G10.6-0.4.

The simplest resolution of this mismatch to which we are forced is, therefore, to reconsider the initial assumption, which was that nitrogen atoms arriving at grain surfaces are all converted to $\mathrm{NH}_{3}$ molecules and injected into the gas-phase. We show in Table 3 our results for the RM in which the nitrogen hydrides ejected into the gas-phase are distributed equally between $\mathrm{NH}, \mathrm{NH}_{2}$, and $\mathrm{NH}_{3}$, rather than all in $\mathrm{NH}_{3}$. With this modification, our calculations (see Table 3, Model A) for the RM but with a reduced RF $\left(0.25 G_{0}\right)$ give ratios $\mathrm{NH}_{3} /$ $\mathrm{NH}$ and $\mathrm{NH}_{3} / \mathrm{NH}_{2}$ of 0.5 and 0.9 , close to observed, and with fractional abundances that are only slightly lower than are observed, a deficiency that can be resolved at a slightly higher density (Table 3, Model B).

Persson et al. (2012) have reanalyzed the nitrogen hydride data of G10.6-0.4, and also present data for foreground diffuse material toward G49N. The column density ratios $N(\mathrm{NH}) / N(\mathrm{o}-$ $\left.\mathrm{NH}_{3}\right)$ and $N\left(\mathrm{o}-\mathrm{NH}_{2}\right) / N\left(\mathrm{o}-\mathrm{NH}_{3}\right)$ over different velocity components are fairly constant at 3.2 and 1.9 , respectively, for $\mathrm{W} 49 \mathrm{~N}$ and 5.4 and 2.2 for G10.6-0.4. The fractional abundance of 

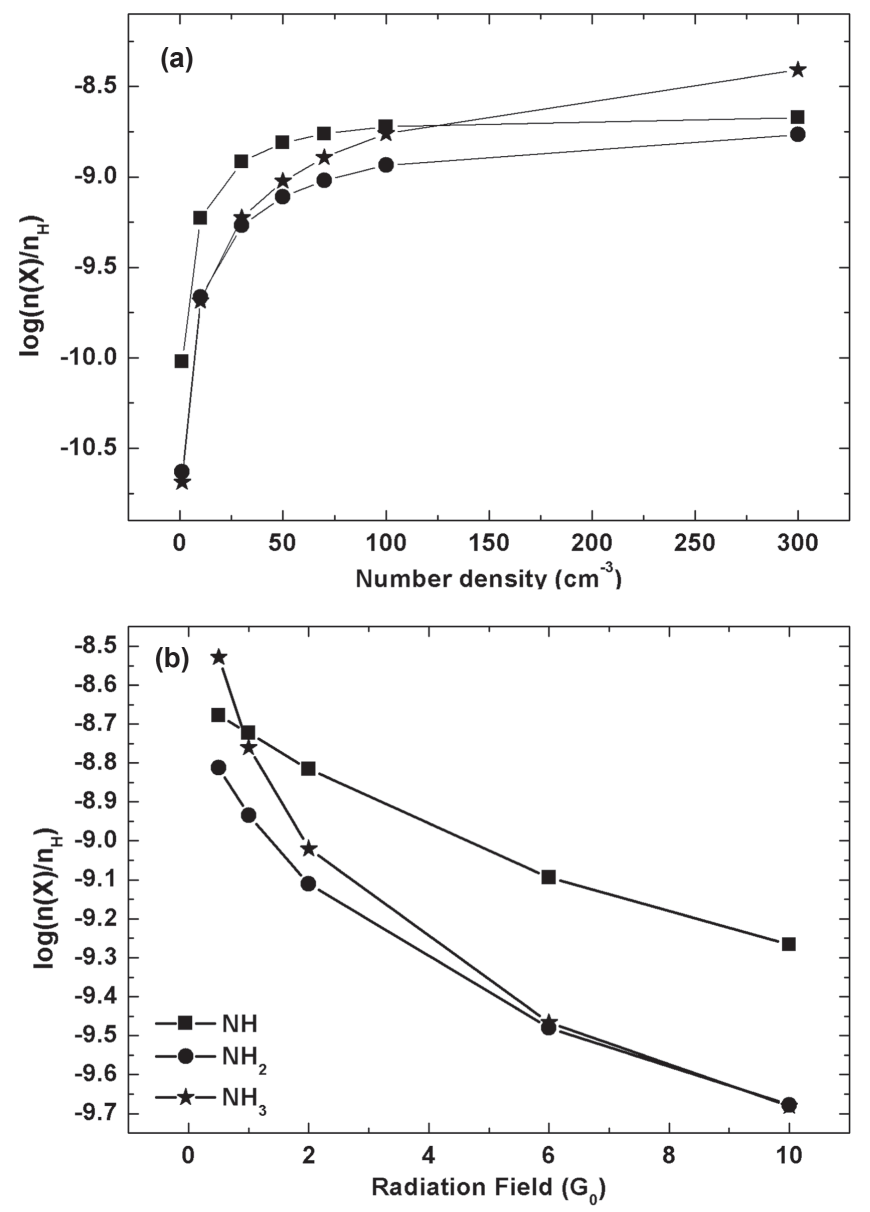

Figure 3. (a) Steady-state fractional abundances of $\mathrm{NH}, \mathrm{NH}_{2}$, and $\mathrm{NH}_{3}$ for number densities between $n_{\mathrm{H}}=1$ and $300 \mathrm{~cm}^{-3}$. (b) As for (a), but for radiation fields $\mathrm{RF}=0.1-10 \times G_{0}$.

o- $\mathrm{NH}_{3}$ is $\sim 2 \times 10^{-9}$ in both sources. These results are better fitted by models such as those shown in Figure $3(\mathrm{~b})$. The material in nitrogen hydrides is $\sim 50 \%$ greater in G10.6-0.4 than in W49N. This variation could be accounted for by either a slightly lower gas density or a larger RF in W49N (see Figures 3(a) and (b)).

\subsection{Nitrogen Hydrides in Dense Gas}

The main purpose of this paper has been to discuss the formation of hydrides of $\mathrm{N}$ atoms and $\mathrm{O}^{+}$ions in diffuse gas, such as that toward G10.6-0.4. However, it is useful to make a comparison with models of chemistry that have been developed to account for the abundances of nitrogen hydrides and their ratios in much denser gas. An exceptionally comprehensive study of the gas-phase chemistry of nitrogen hydrides in dark clouds has been carried out by Le Gal et al. (2014a). The chemical network used in the present study is similar to but significantly larger than that of Le Gal et al., but that study is very much more detailed in several important respects. In our study, the effects of the spin symmetries of the nitrogen hydrides and the ortho-para forms of molecular hydrogen have been ignored, whereas Le Gal et al. have determined, selfconsistently, their role in the chemistry. Le Gal et al. have also demonstrated that the amounts of carbon, oxygen, and sulfur available in the gas have significant effects on the abundances of nitrogen hydrides. These occur through the enhancement of
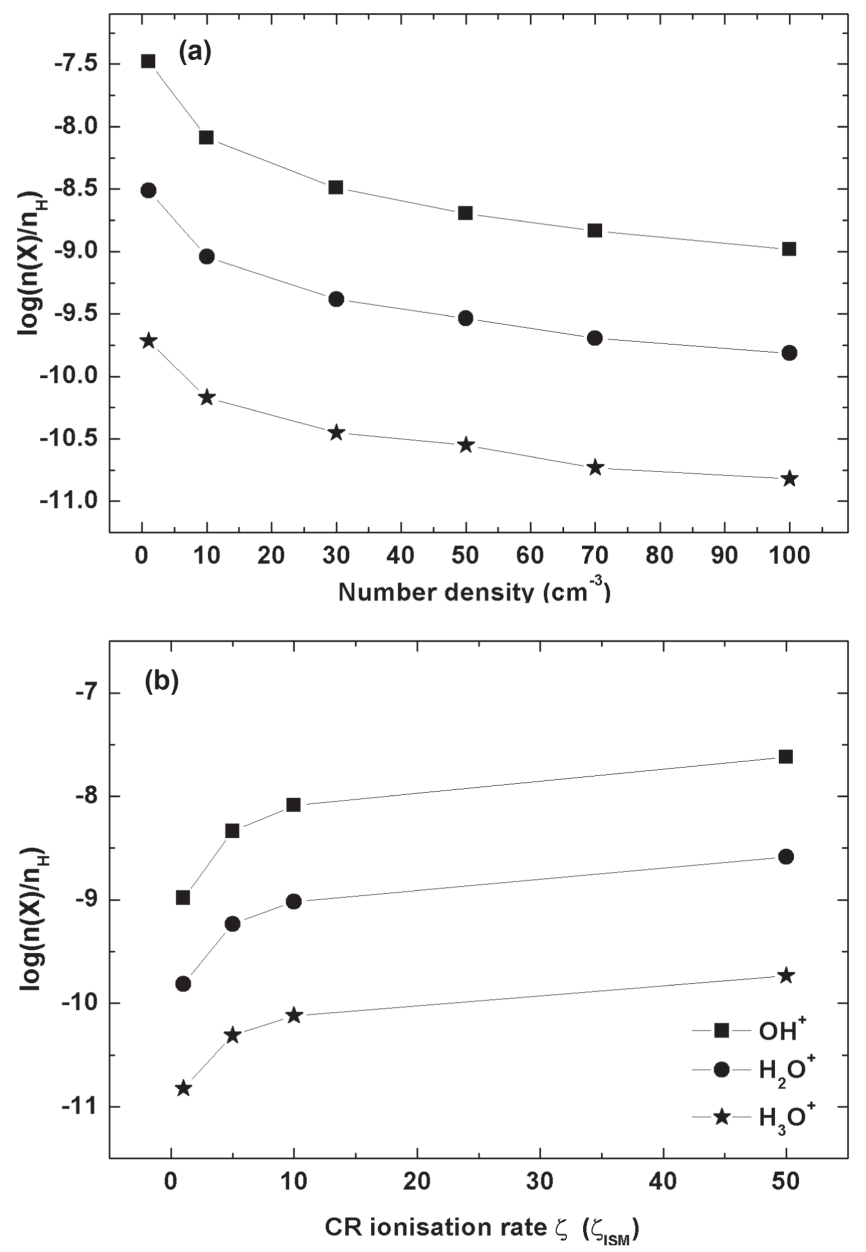

Figure 4. As for Figure 3, but for $\mathrm{OH}^{+}, \mathrm{H}_{2} \mathrm{O}^{+}$, and $\mathrm{H}_{3} \mathrm{O}^{+}$, with fractional abundances at peak $\mathrm{OH}^{+}$. Panel (a) is for cloud number densities $n_{\mathrm{H}}=1-100 \mathrm{~cm}^{-3}$; panel (b) is for cosmic-ray fluxes $1-50 \times \zeta_{\text {ISM }}$.

$\mathrm{N}_{2}$ through neutral chemistries such as $\mathrm{N}+\mathrm{CH} \longrightarrow \mathrm{CN}+\mathrm{H}$ followed by $\mathrm{CN}+\mathrm{N} \longrightarrow \mathrm{C}+\mathrm{N}_{2}$, and $\mathrm{OH}+\mathrm{N} \longrightarrow \mathrm{NO}+\mathrm{H}$ followed by $\mathrm{NO}+\mathrm{N} \longrightarrow \mathrm{N}_{2}+\mathrm{O}$. The role of sulfur, as $\mathrm{S}^{+}$, is to destroy $\mathrm{CH}$ and (marginally) $\mathrm{OH}$, and therefore inhibit the growth of $\mathrm{N}_{2}$. All these reactions are included in the network of the present paper, and so the general behavior of chemistry is similar in both models, even though the effects of spin symmetries and $\mathrm{H}_{2}$ ortho/para reactions have been ignored in our work. Given sufficient time (typically, several million years) gas-phase reactions convert most of the available nitrogen to $\mathrm{N}_{2}$ molecules, and reactions $\mathrm{N}_{2}+\mathrm{H}_{3}^{+} \longrightarrow \mathrm{N}_{2} \mathrm{H}^{+}$ $+\mathrm{H}_{2}$ followed by the dissociative recombination of $\mathrm{N}_{2} \mathrm{H}^{+}$ (discussed in Section 3 above) become the major source of $\mathrm{NH}$ and hence of other nitrogen hydrides.

Le Gal et al. (2014b) have compared the predictions of their models with observational results obtained by Herschel/HIFI of $\mathrm{NH}, \mathrm{NH}_{2}$, and $\mathrm{NH}_{3}$ detections in the envelope of the protostar IRAS 16293-2422. They also reassessed earlier determinations of the hydride abundances by Hily-Blant et al. (2010) and obtained the ratio $\mathrm{NH}: \mathrm{NH}_{2}: \mathrm{NH}_{3}=3: 1: 19$, in a cloud in which $N_{\mathrm{H}}=(3.0 \pm 1.5) \times 10^{22} \mathrm{~cm}^{-2}$. Le Gal et al. find that their model gives good fits to both abundances and ratios of nitrogen hydrides with specific choices for $\mathrm{C} / \mathrm{O}$ and $\mathrm{S}$ abundances. 
Table 2

Fractional Abundances and Ratios at Different Number Density and Radiation Field for Nitrogen Hydrides

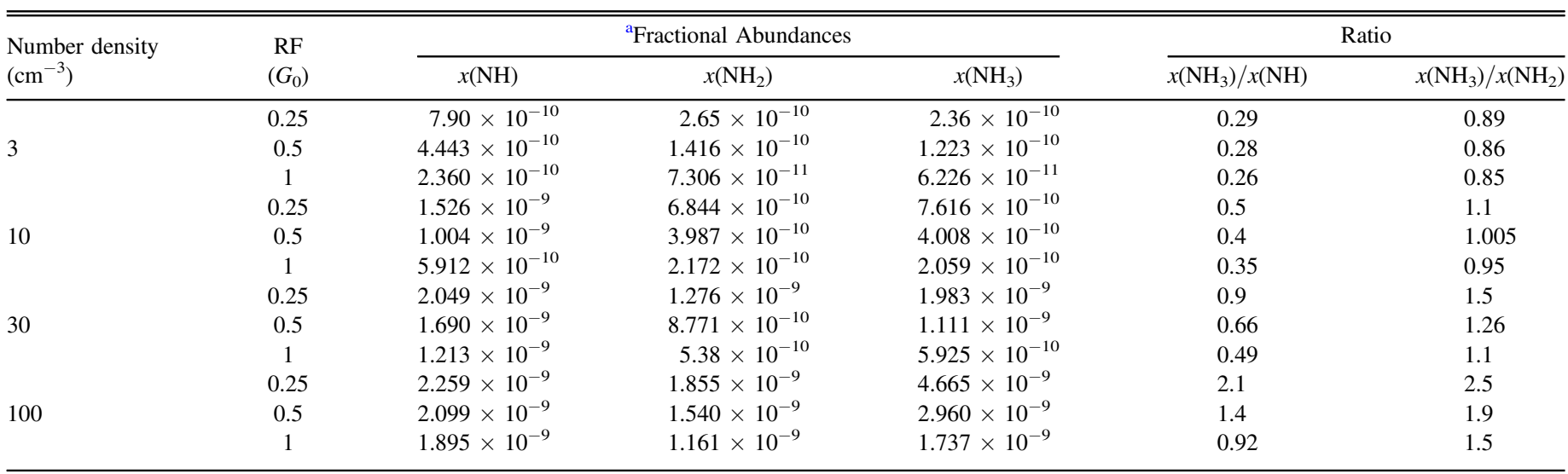

Note.

${ }^{\text {a }}$ The fractional abundances, $x(\mathrm{Y})$, are defined by $n(\mathrm{Y}) / n_{\mathrm{H}}$.

Table 3

Fractional Abundances and Ratios for the RM When N-hydrides are Formed in Equal (Model A) or Varying Proportions (Model B) on Grain Surfaces

\begin{tabular}{|c|c|c|c|c|c|c|c|}
\hline \multirow{2}{*}{$\begin{array}{l}\text { Density } \\
\left(\mathrm{cm}^{-3}\right)\end{array}$} & \multirow{2}{*}{$\begin{array}{l}\mathrm{RF} \\
\left(G_{0}\right)\end{array}$} & \multirow{2}{*}{ Model } & \multicolumn{3}{|c|}{${ }^{\mathrm{a}}$ Fractional Abundances } & \multicolumn{2}{|c|}{ Ratio } \\
\hline & & & $x(\mathrm{NH})$ & $x\left(\mathrm{NH}_{2}\right)$ & $x\left(\mathrm{NH}_{3}\right)$ & $x\left(\mathrm{NH}_{3}\right) / x(\mathrm{NH})$ & $x\left(\mathrm{NH}_{3}\right) / x\left(\mathrm{NH}_{2}\right)$ \\
\hline \multirow[t]{2}{*}{100} & 0.25 & A & $2.654 \times 10^{-9}$ & $1.631 \times 10^{-9}$ & $1.439 \times 10^{-9}$ & 0.54 & 0.88 \\
\hline & & B & $3.120 \times 10^{-9}$ & $1.307 \times 10^{-9}$ & $9.332 \times 10^{-10}$ & 0.29 & 0.71 \\
\hline & & B & $3.490 \times 10^{-9}$ & $1.582 \times 10^{-9}$ & $1.384 \times 10^{-9}$ & 0.39 & 0.87 \\
\hline
\end{tabular}

Note. The results are shown for the $\mathrm{RM}$ in the case when surface reactions provide $\mathrm{NH}, \mathrm{NH}_{2}$, and $\mathrm{NH}_{3}$ either in equal proportions (Model A) or in varying proportions of $0.5,0.3,0.2$, respectively (Model B).

a The fractional abundances, $x(\mathrm{Y})$, are defined by $n(\mathrm{Y}) / n_{\mathrm{H}}$.

We come to a similar conclusion for our model with more extensive but less detailed gas-phase chemistry. Without detailed fitting, our purely gas-phase chemistry for a cloud with density $n_{\mathrm{H}}=10^{4} \mathrm{~cm}^{-3}$ and $A_{\mathrm{V}}=3 \mathrm{mag}$ leads to the nitrogen hydride ratio $2: 1: 8$, reasonably similar to the revised observational values. Our model for gas-phase + grain surface chemistries gives a much worse fit to the ratio.

Thus, it appears that the role for surface reactions in determining nitrogen hydride abundances is clear in diffuse clouds, but not in dense dark clouds. In dark clouds, the high abundance of $\mathrm{N}_{2}$ enables the opening-up of an additional and efficient gas-phase channel leading to nitrogen hydrides.

The situation in star-forming regions is, of course, more complicated than in quiescent dark clouds. The timescale for freeze-out in clouds of number density $n_{\mathrm{H}}=10^{4} \mathrm{~cm}^{-3}$ is much less than the likely cloud age. Molecules are very likely to visit a grain surface and may make such visits many times, depending on the timescale for desorption. Thus, the simple picture presented here may, in fact, be more complicated. For example, models of complex chemistry, such as that of Garrod et al. (2008) assume that $\mathrm{N}, \mathrm{NH}$, and $\mathrm{NH}_{2}$ are hydrogenated in grain-surface chemistry in regions of star formation. Such processes would affect the hydride abundances and ratios.

\subsection{Hydrides of Oxygen Ions}

There is a wealth of observational data on the hydrides of $\mathrm{O}^{+}$ observed on 20 Galactic lines of sight toward bright submillimeter continuum sources (Indriolo et al. 2015). On each line of sight, a number (from 2 to 13) of distinct velocity components are found, so that the total number of sources is over 100. Positive detections of the ion $\mathrm{H}_{3} \mathrm{O}^{+}$were made on seven of the 20 lines of sight and 16 of the velocity components (most of these toward Sgr B2 (M and N) where the ions $\mathrm{OH}^{+}$ and $\mathrm{H}_{2} \mathrm{O}^{+}$are missing. Ignoring $\mathrm{Sgr} \mathrm{B} 2, \mathrm{H}_{3} \mathrm{O}^{+}$is detected in only six of the components in which $\mathrm{OH}^{+}$and $\mathrm{H}_{2} \mathrm{O}^{+}$are present. Apart from Sgr B2, $\mathrm{OH}^{+}$and $\mathrm{H}_{2} \mathrm{O}^{+}$are found together in almost all velocity components. The ratio of column densities $N\left(\mathrm{OH}^{+}\right) / N\left(\mathrm{H}_{2} \mathrm{O}^{+}\right)$typically lies in the range 1-10, with very few outliers.

Column densities for $\mathrm{OH}^{+}, \mathrm{H}_{2} \mathrm{O}^{+}$, and atomic $\mathrm{H}$ are provided where possible by Indriolo et al. (2015). These authors infer that the fraction of hydrogen as $\mathrm{H}_{2}$ is generally small, about a few per cent. The fractional abundances of $\mathrm{OH}^{+}$ typically lie in the range $10^{-9}$ to $\sim 3 \times 10^{-8}$.

Figures 4(a) and (b) show how the computed fractional abundances vary with number density and cosmic-ray ionization rate. These fractional abundances are taken at the epoch at which peak $\mathrm{OH}^{+}$fractional abundance occurs. It is evident that low densities and high cosmic-ray ionization rates give the largest fractional abundances, and that these are at the upper end of the observed range. As noted above, such low-density clouds are unlikely to attain a chemical steady state within the lifetime of the clouds, and therefore peak fractional abundances are more appropriate to consider than steady-state values. Our results show that peak $\mathrm{OH}^{+}$fractional abundances in the observed range of about $1 \times 10^{-9}$ to $3 \times 10^{-8}$ are obtained, either with cloud number densities of the order of $\sim 10 \mathrm{~cm}^{-3}$ and a canonical cosmic-ray ionization rate, or in a cloud with a somewhat higher 
Table 4

The Fractional Abundances and Ratios at Different Number Densities and CR Ionization Rates for $\mathrm{O}$ and $\mathrm{O}^{+}$Hydrides

\begin{tabular}{|c|c|c|c|c|c|c|}
\hline \multirow{2}{*}{$\begin{array}{l}\text { Number density } \\
\left(\mathrm{cm}^{-3}\right)\end{array}$} & \multirow{2}{*}{$\zeta / \zeta_{\text {ISM }}$} & \multicolumn{4}{|c|}{${ }^{\mathrm{a}}$ Fractional Abundances } & \multirow{2}{*}{$\begin{array}{c}\text { Ratio } \\
x\left(\mathrm{OH}^{+}\right) / x\left(\mathrm{H}_{2} \mathrm{O}^{+}\right)\end{array}$} \\
\hline & & $x(\mathrm{OH})$ & $x\left(\mathrm{H}_{2} \mathrm{O}\right)$ & $x\left(\mathrm{OH}^{+}\right)$ & $x\left(\mathrm{H}_{2} \mathrm{O}^{+}\right)$ & \\
\hline \multirow[t]{2}{*}{100} & 50 & $1.786 \times 10^{-7}$ & $4.677 \times 10^{-9}$ & $2.397 \times 10^{-8}$ & $2.589 \times 10^{-9}$ & $9.258^{\mathbf{p}}$ \\
\hline & & $5.736 \times 10^{-7}$ & $3.743 \times 10^{-8}$ & $1.711 \times 10^{-9}$ & $1.435 \times 10^{-9}$ & $1.192^{\mathrm{ss}}$ \\
\hline \multirow[t]{2}{*}{10} & 1.0 & $2.767 \times 10^{-8}$ & $2.085 \times 10^{-9}$ & $8.074 \times 10^{-9}$ & $8.732 \times 10^{-10}$ & $9.246^{\mathbf{p}}$ \\
\hline & & $3.089 \times 10^{-8}$ & $3.770 \times 10^{-9}$ & $2.732 \times 10^{-10}$ & $2.374 \times 10^{-10}$ & $1.151^{\mathrm{ss}}$ \\
\hline
\end{tabular}

Note. The results are shown at the peak position of $\mathrm{OH}^{+}$, "p", and at steady-state "ss".

${ }^{\text {a }}$ The fractional abundances, $x(\mathrm{Y})$, are defined by $n(\mathrm{Y}) / n_{\mathrm{H}}$.

density, $\sim 100 \mathrm{~cm}^{-3}$, but with a greatly enhanced cosmic-ray rate, $\sim 50 \times \zeta_{\text {ISM. }}$. The computed $\mathrm{H}_{2} \mathrm{O}^{+}$fractional abundance (taken at peak $\mathrm{OH}^{+}$) is generally an order of magnitude less than that of $\mathrm{OH}^{+}$(consistent with observations), and $\mathrm{H}_{3} \mathrm{O}^{+}$is even less abundant. Ratios $\mathrm{OH}^{+} / \mathrm{H}_{2} \mathrm{O}^{+}$closer to unity can be obtained when the chemistry is nearer steady-state, rather than at peak $\mathrm{OH}^{+}$. However, these fractional abundances are low, in absolute terms, and a high cosmic-ray flux would be required to raise them into the observed range. These results appear to be consistent with the observational data of Indriolo et al. (2015). The solution for the $\mathrm{O}^{+}$hydrides based on very high cosmic-ray ionization rates would also imply high fractional abundances of $\mathrm{OH}$ and $\mathrm{H}_{2} \mathrm{O}$ (see Table 4).

\subsection{Conclusions}

While conventional gas-phase chemistry in diffuse clouds can account for the detected fractional abundances of hydrides of $\mathrm{O}^{+}$, surface reactions contributing $\mathrm{O}$ atom hydrides to the gas make little difference to the fractional abundances of $\mathrm{O}^{+}$ ions and only marginal differences to the fractional abundances of neutrals $\mathrm{OH}$ and $\mathrm{H}_{2} \mathrm{O}$. The values of detected fractional abundances of the $\mathrm{O}^{+}$hydrides indicate that these species may be found either in conventional diffuse clouds $\left(n_{\mathrm{H}} \sim 100 \mathrm{~cm}^{-3}\right)$ with very high cosmic-ray ionization rates or in very lowdensity diffuse clouds with normal cosmic-ray fluxes.

On the other hand, the nitrogen hydrides detected in lowdensity gas cannot be formed in the observed fractional abundances by gas-phase reactions, and surface reactions are required to form these hydrides, with an efficiency similar to that of $\mathrm{H}_{2}$ formation. The location of these hydrides is indicated by these models to be fairly conventional diffuse clouds. The observational results (Persson et al. 2012) suggest that surface reactions inject not only $\mathrm{NH}_{3}$ molecules but also $\mathrm{NH}_{2}$ and $\mathrm{NH}$ radicals. Evidently, surface reactions may be important in diffuse cloud chemistry, and their contribution in gas-phase models of diffuse clouds should be assessed. However, hydride chemistry in dense dark clouds appears to be determined by gas-phase chemistry.

We thank the referees for comments that helped to improve an earlier version of the manuscript. Z.A. thanks the Egyptian Science and Technology Development Funds, STDF, for funding the research leading to these results through the STF-Cycle 5/2014-2015 under project ID: 12334. S.V. acknowledges support from an STFC consolidated grant (grant number ST/M001334/1).

\section{REFERENCES}

Asplund, M., Grevesse, N., Sauval, A. J., \& Scott, P. 2009, ARA\&A, 47, 481

Bayet, E., Williams, D. A., Hartquist, T. W., \& Viti, S. 2011, MNRAS, 414, 1583

Crawford, I. A., \& Williams, D. A. 1997, MNRAS, 291, L53

Dislaire, V., Hily-Blant, P., Faure, A., et al. 2012, A\&A, 537, A20

Draine, B. T. 2011, Physics of the Interstellar and Intergalactic Medium (Princeton, NJ: Princeton Univ. Press)

Fedoseev, G., Ioppolo, S., Zhao, D., Lamberts, T., \& Linnartz, H. 2015, MNRAS, 446, 439

Garrod, R. T., Weaver, S. L. W., \& Herbst, E. 2008, ApJ, 682, 283

Gerin, M., de Luca, M., Black, J., et al. 2010, A\&A, 518, L110

Gerlich, D. 1993, J. Chem. Soc., Faraday Trans., 89, 2199

Gupta, H., Rimmer, P., Pearson, J. C., et al. 2010, A\&A, 521, L47

Hidaka, H., Watanabe, M., Kouchi, A., \& Watanabe, N. 2011, PCCP, 13, 15798

Hily-Blant, P., Maret, S., Bacmann, A., et al. 2010, A\&A, 521, L52

Hiraoka, K., Yamashita, A., Yachi, Y., et al. 1995, ApJ, 443, 363

Hollenbach, D., Kaufman, M. J., Neufeld, D., Wolfire, M., \& Goicoechea, J. R. 2012, ApJ, 754, 105

Indriolo, N., Neufeld, D. A., Gerin, M., et al. 2015, ApJ, 800, 40

Jura, M. 1974, ApJ, 191, 375

Knauth, D. C., Andersson, B.-G., McCandliss, S. R., \& Warren Moos, H. 2004, Natur, 429, 636

Le Bourlot, J. 1991, A\&A, 242, 235

Le Gal, R., Hily-Blant, P., Faure, A., et al. 2014a, A\&A, 562, A83

Le Gal, R., Hily-Blant, P., \& Faure, A. 2014b, in SF2A-2014: Proc. Annual Meeting of the French Society of Astronomy and Astrophysics, ed. J. Ballet et al., 397

Le Petit, F., Roueff, E., \& Herbst, E. 2004, A\&A, 417, 993

Le Teuff, Y. H., Millar, T. J., \& Markwick, A. J. 2000, A\&AS, 146, 157

Linnartz, H., Ioppolo, S., \& Fedoseev, G. 2015, IRPC, 34, 205

Marquette, J. B., Rebrion, C., \& Rowe, B. R. 1988, JChPh, 89, 2041

McCrea, W. H., \& McNally, D. 1960, MNRAS, 121, 238

McElroy, D., Walsh, C., Markwick, A. J., et al. 2013, A\&A, 550, A36

Meyer, D. M., \& Roth, K. C. 1991, ApJ, 376, L49

Millar, T. J., Farquhar, P. R. A., \& Willacy, K. 1997, A\&AS, 121, 139

Persson, C. M., Black, J. H., Cernicharo, J., et al. 2010, A\&A, 521, L45

Persson, C. M., De Luca, M., Mookerjea, B., et al. 2012, A\&A, 543, A145

Pickles, J. B., \& Williams, D. A. 1977, Ap\&SS, 52, 443

Roberts, J. F., Rawlings, J. M. C., Viti, S., \& Williams, D. A. 2007, MNRAS, 382,733

Tielens, A. G. G. M. 2005, The Physics and Chemistry of the Interstellar Medium (Cambridge: Cambridge Univ. Press)

Viti, S., Collings, M. P., Dever, J. W., McCoustra, M. R. S., \& Williams, D. A. 2004, MNRAS, 354, 1141

Viti, S., \& Williams, D. A. 1999, MNRAS, 305, 755

Wagenblast, R., \& Williams, D. A. 1996, Ap\&SS, 236, 257

Wagenblast, R., Williams, D. A., Millar, T. J., \& Nejad, L. A. M. 1993, MNRAS, 260, 420

Wakelam, V., Selsis, F., Herbst, E., \& Caselli, P. 2005, A\&A, 444, 883

Woodall, J., Agúndez, M., Markwick-Kemper, A. J., \& Millar, T. J. 2007, A\&A, 466, 1197

Zymak, I., Hejduk, M., Mulin, D., et al. 2013, ApJ, 768, 86 